\title{
Comparison of the Effectiveness of Two Religious-Spiritual and Hope Interventions on Reducing Stress, Anxiety and Depression of Spouses of Veterans with PTSD
}

\section{ART ICLE INF O}

\section{Article Type}

Original Research

\section{Authors}

Taghavi M.*1 $M A$,

Hashemian K. ${ }^{1} P h D$,

Bolhari J. ${ }^{2} M D$

How to cite this article
Taghavi M, Hashemian K, Bolhari
J. Comparison of the Effectiveness
of Two Religious-Spiritual and
Hope Interventions on Reducing
Stress, Anxiety and Depression of
Spouses of Veterans with PTSD.
Iranian Journal of War \& Public
Health.2020;12(3):165-172.

${ }^{1}$ Department of Psychology, Faculty of Psychology and Social Sciences, Roudehen Branch, Islamic Azad University, Roudehen, Iran

${ }^{2}$ Spiritual Health Research Center, School of Behavioral Sciences and Mental Health, Iran University of Medical Sciences, Tehran, Iran

\section{*Correspondence}

Address: University Complex of Islamic Azad University, Roudehen, Iran. Postal Code: 3973188981. Phone: +98 (21) 76506887 Fax: $+98(21) 76507639$ m.taghavi125@gmail.com

\section{Article History}

Received: August 04, 2020

Accepted: January 05, 2021

ePublished: March 14, 2021

\section{A B S T R A C T}

Aims The present study aimed to compare the effects of religious-spiritual intervention and hope therapy on reducing stress, anxiety, and depression of spouses of veterans with the post-traumatic stress disorder (PTSD).

Materials \& Methods Using the pretest-posttest design with a control group and follow-up, this quasi-experimental study was conducted among spouses of veterans who had referred to Sadr hospital in Tehran since 2003 to 2016. The participants were randomly assigned into two experimental groups and one control group (each group 17 persons). The data were collected using the Secondary Traumatic Stress Scale (STSS-17) and Depression, Anxiety, Stress Scale (DASS-21). The experimental groups 1 and 2 were trained three times a week in eight sessions. A week after termination of training sessions, posttest was administered for the two experimental groups and the control group. Three months after termination of training program, participants were tested again to follow up the training effects. To compare the effectiveness of the approaches, two-way multivariate analysis of variance (MANOVA) mixed design was used.

Findings Both religious-spiritual and hope interventions significantly reduced stress, anxiety, and depression of spouses of veterans with PTSD compared to the control group and also the effects have been stable after a three-month follow-up $(\mathrm{p}<0.01)$. Whereas, there was no significant difference between the effectiveness of these two interventions on reducing stress, anxiety, and depression of spouses of veterans compared to the control group ( $p>0.05)$. Conclusion The two religious-spiritual and hope approaches are effective as interactive interventions on reducing stress, anxiety, and depression of spouses of veterans with PTSD, and there is no reference between them.

Keywords Spirituality; Religion; Hope; Psychological Stress; Anxiety; Depression

\section{I T A T I O N L I N KS}

[1] Comparison of the effectiveness ... [2] Diagnostic and statistical ... [3] Psychological sequelae of combat ... [4] Secondary traumatic stress ... [5] Psychiatric and health impact of primary ... [6] The relationship between acute ... [7] Post-traumatic stress disorder and ... [8] Secondary traumatization among ... [9] Caregiver burden and ... [10] National guard families ... [11] Comparison of depression, anxiety ... [12] Marital intimacy, family ... [13] The relationship between personality ... [14] A systemic review of interventions ... [15] Comparison of the effectiveness ... [16] The effectiveness of instruction ... [17] Development and validation ... [18] Investigate of validity and reliability ... [19] The structure of negative ... [20] Reliability and validity of ... [21] Psychiatry and man's ... [22] Logotherapy and existentialism ... [23] Casebook for a spiritual ... [24] Target Article: Hope ... [25] Hand book of hope ... [26] Strategic hopefocused relationship ... [27] Examining the links ... [28] A spirituality teaching ... [29] The effectiveness of ... [30] Effect of spiritual group ... [31] Using spiritual interventions ... [32] Compiling and evaluating ... [33] Spiritual healing ... [34] Hope and depression ... [35] You have got hope, studies ... [36] Hope therapy in a Community ... [37] The effectiveness of hope ... [38] Religious psychotherapy in ... [39] Blessed assurance: Religion ... [40] The comparison of ... [41] Meaning therapy: An ... [42] Psychotherapy and ... [43] Hope as a predictor of anxiety ... [44] Hope conquers fear ... [45] Interaction of hope ... [46] The effects of optimism ... [47] Examining hope as a trans ... [48] An ecological view of ... [49] Hope for rehabilitation ... [50] Hope therapy: Helping ... [51] Patterns of positive and negative ... [52] The role of spirituality ... [53] Religious coping among ... [54] Forgiveness is an ... [55] Relationship among forgiveness ... [56] Handbook of positive ... [57] A longitudinal study ...[58] Hope as a source of resilience ... 
با انواع اختلالات روانى از جمله اختلال استرس يس از ضربه همراه بوده كه اله

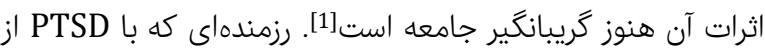

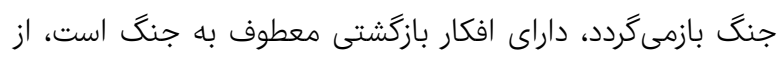

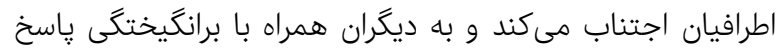

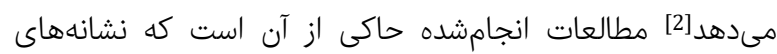

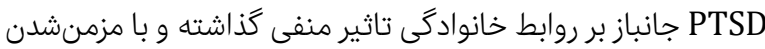

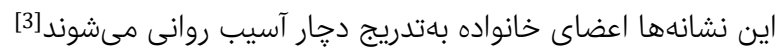
و بهسبب تماس نزديك با فردى كه قربانى مستقيم تروما است، آن آن

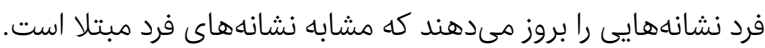

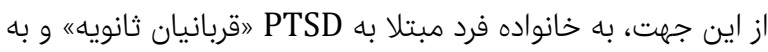
اختلال ايجادشده تروماى ثانويه Secondary Traumatic) Stress Disorder; STSD)

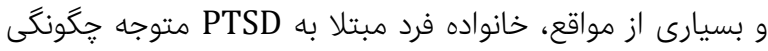

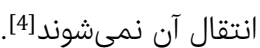
يزووهشهاى انجامشده حاكى از آن است كه همسران جانبازان مبتلا

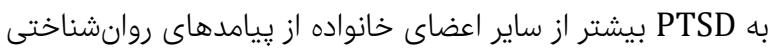

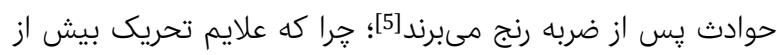
حد جانبازان موجب مىشود كه همسرانشان در معرض آسريب آسيبهاى

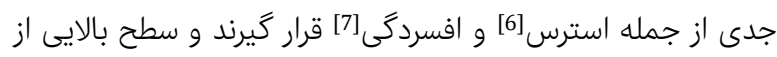

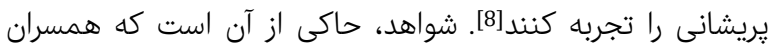

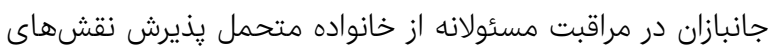

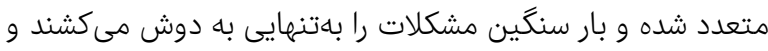

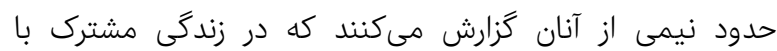
شوهرانشان از با درآمدهاند [9].

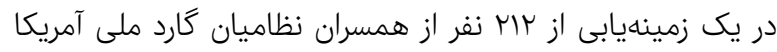

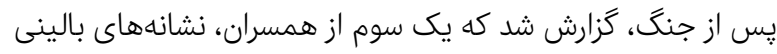

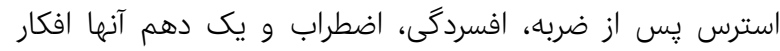

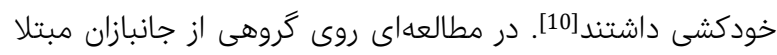

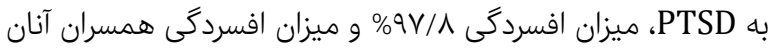

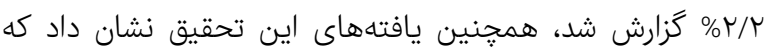

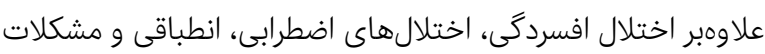

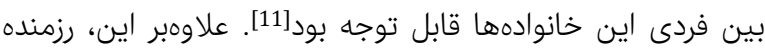

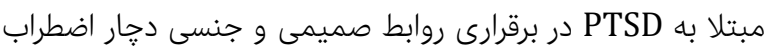

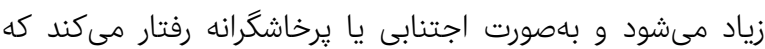

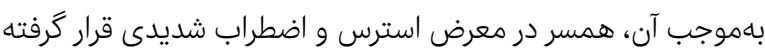

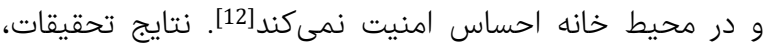

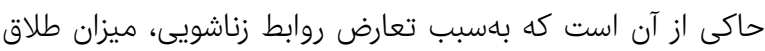

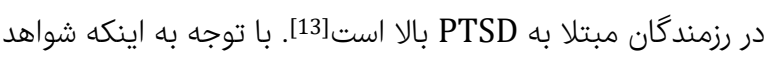

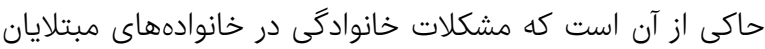

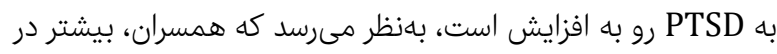

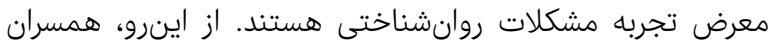

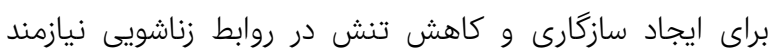

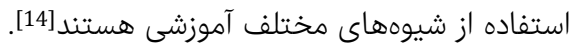

مقايسه اثربخشى دو مداخله معنوى-مذهبى و

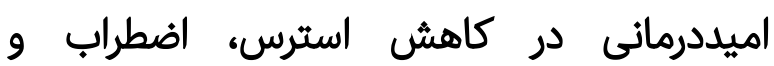
افسردگى همسران جانبازان مبتلا به استرس یس از ضربه

MA منيره تقوى كروه روانشناسى، دانشكده روانشناسى و علوم اجتماعى، واحد رودهن، دانشكاه آزاد اسلامى، رودهن، ايران

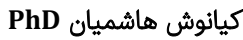

كَروه روانشناسى، دانشكده روانشناسى و علوم اجتماعى، واحد رودهن، دانشكاه آزاد اسلامى، رودهن، ايران

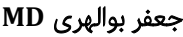
مركز تحقيقات سلامت معنوى، دانشكده علوم رفتارى و سلامت روان، دانشكاه علوم يزشكى ايران، تهران، ايران

جكيده اهداف: هدف از يزوهش حاضر مقايسه اثر دو مداخله معنوى-مذهبى و

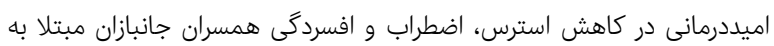

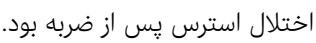

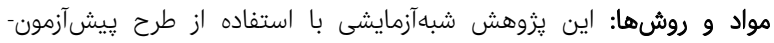

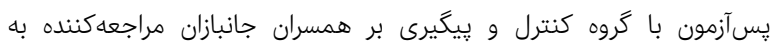

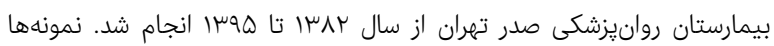

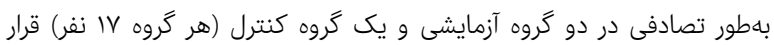

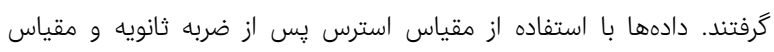

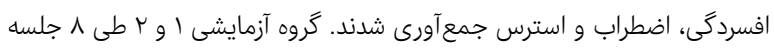

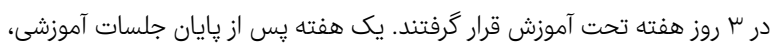

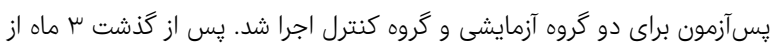

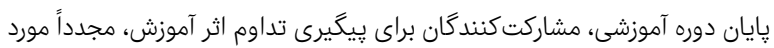

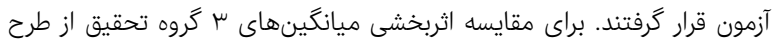

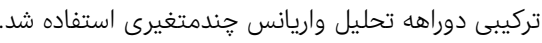

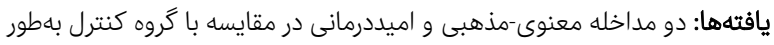

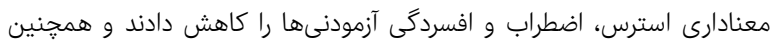

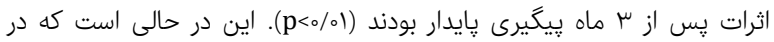

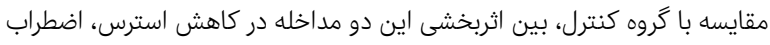

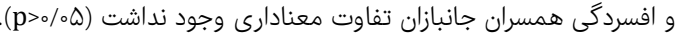
نتيجه

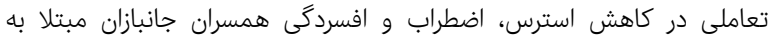
استرس يس از ضربه موثرند و تفاوتى ندارند.

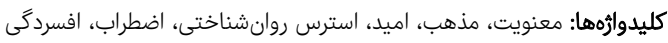

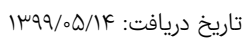

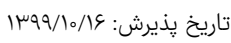
m.taghavi125@gmail.com : نويسنده مسئول: بارئرش

مقدمه - مقد

جنگ بهُعنوان عامل استرسزاى روانى، مشكلات فراوان و غيرقابل

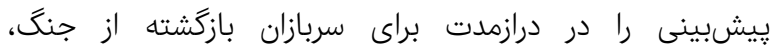
خانوادههايشان و حتى جامعه بهدنبال دارد. جنَّ تحميلى لرساله

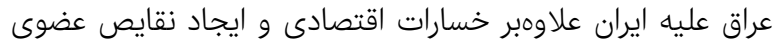




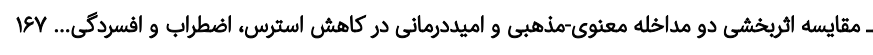

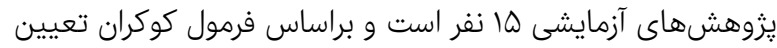

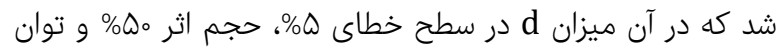

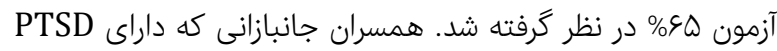
بودند، تمايل به شركت در تحقيق داشتند، سطح تحصيلاتشان

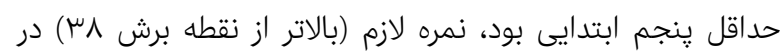

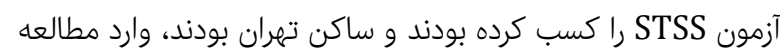

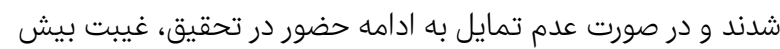

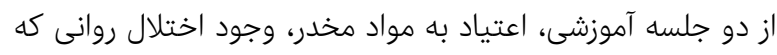

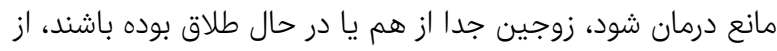
تحقيق كنار گذاشته شدند.

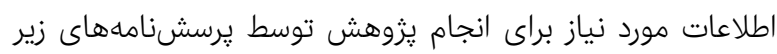

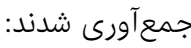

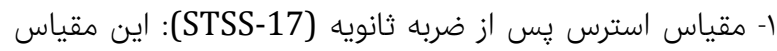

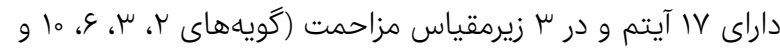

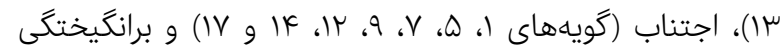

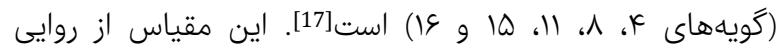

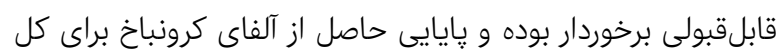

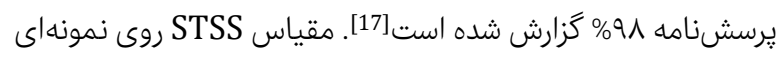

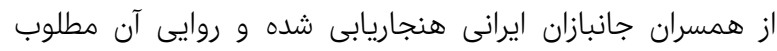

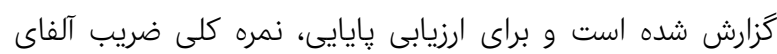
كرونباخ \\\% بهدست آمده است [18].

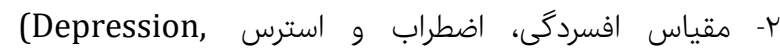
Anxiety, Stress Scale; DASS-21)

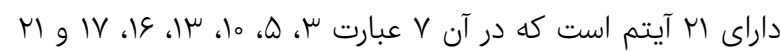

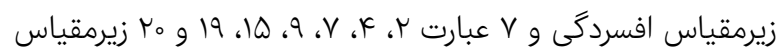

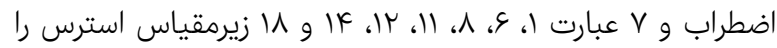

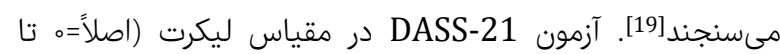

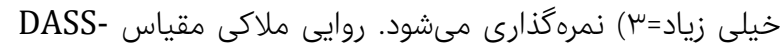

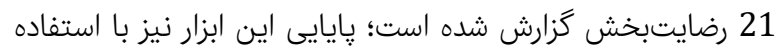

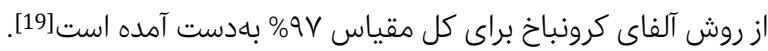
در ايران، فرم كوتاه

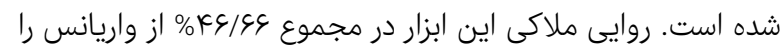

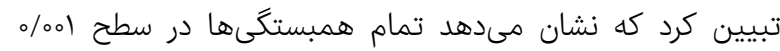

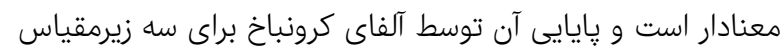

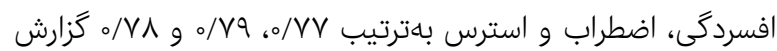
شد

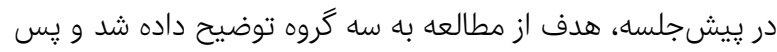

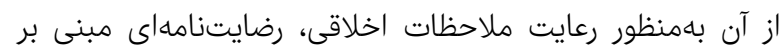

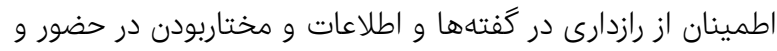
خروج از جلسات آموزشى از همسران كَرفته شد. در ادادامه، گَروهها

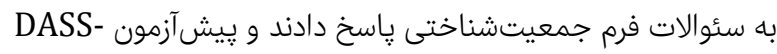

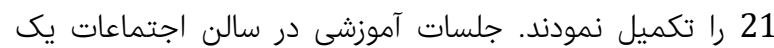

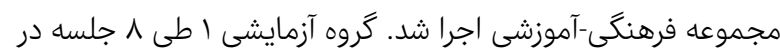

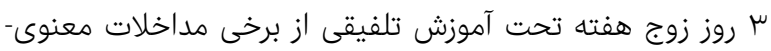

اكرجه مشكلات همسران جانبازان مبتلا به PTSD غيرقابل اجتناب

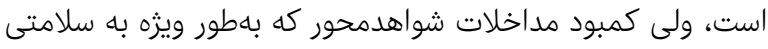

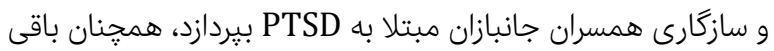

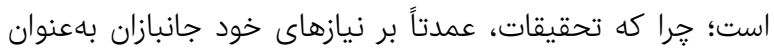

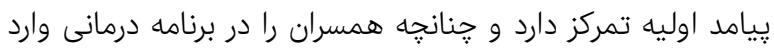

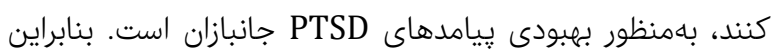

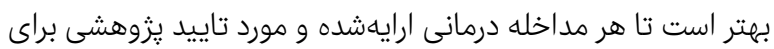

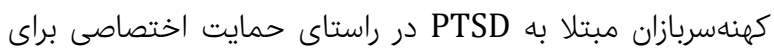

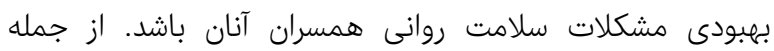

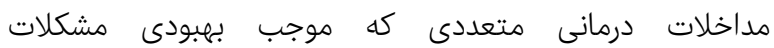
روانشناختى همسران شدهاند، تحقيقى است كه با دو دو راهبرد

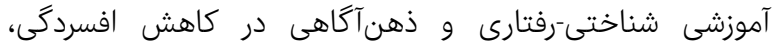

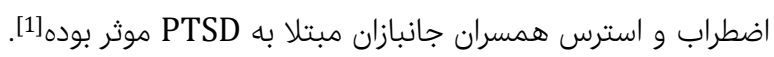

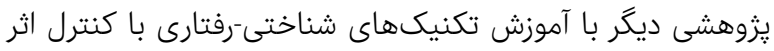

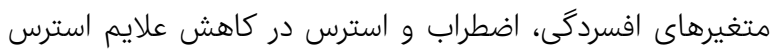

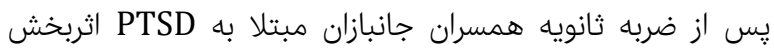

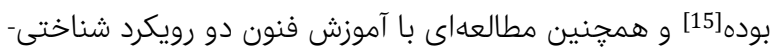

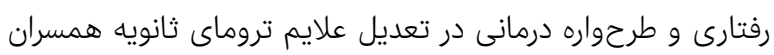

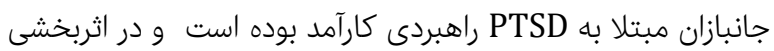
تفاوتى با هم نداشتند [16].

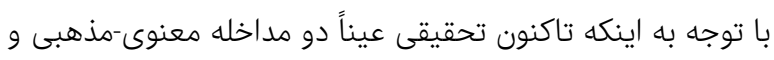

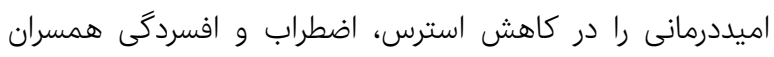

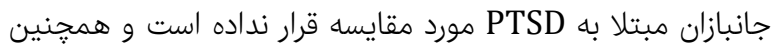

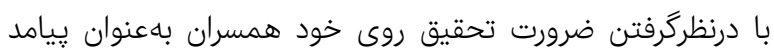

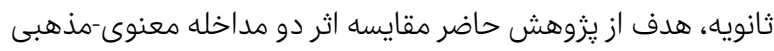
و اميددرمانى در كاهش استرس، اضطراب و إنسر افسردكى همسران

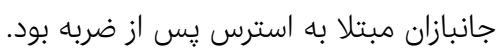

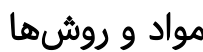

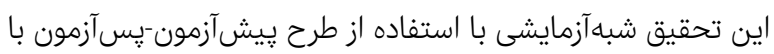

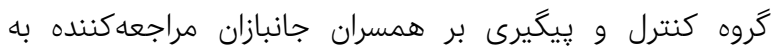

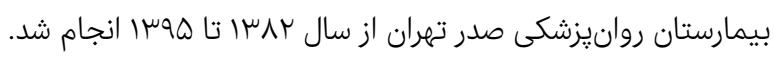

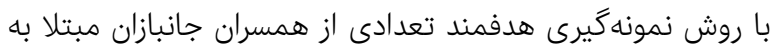

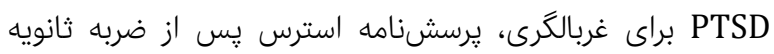
ا) (Secondary Traumatic Stress Scale; STSS-17)

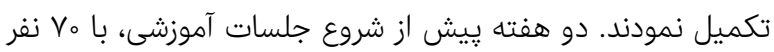

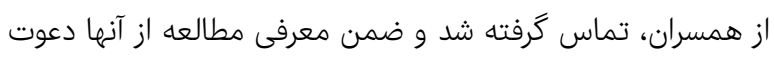

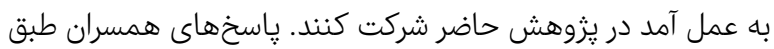

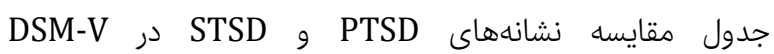
(Diagnostic and Statistical Manual of Mental [2] Disorders)

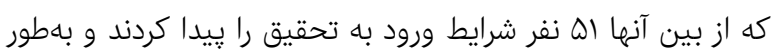

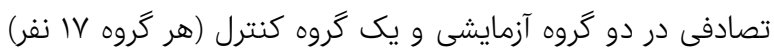

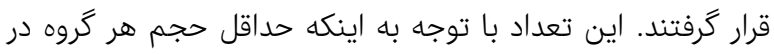


آموزش، مجدداً مورد آزمون قرار گرفتند. گروه كنترل در اين دوره هيج كونه آموزشى را دريافت نكردند. دادهها در دو بخش توصيفى و استنباطى ارزيابى شدند. درياف در بخش درئ

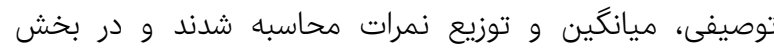

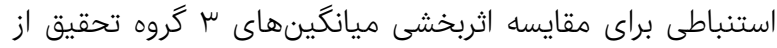

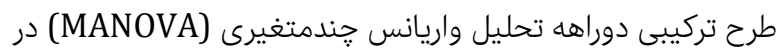
قالب نرمافزار SPSS 20 SPS استفاده شد.

\section{يافتهها}

ميانكين سن شركت كنندكان گروه آزمايشى اول (معنوى-مذهبى)

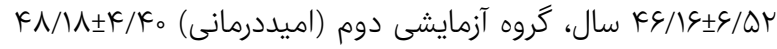
سال و گروه كنترل

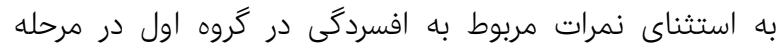

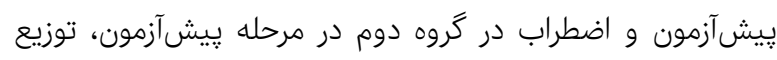

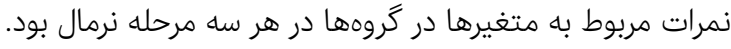

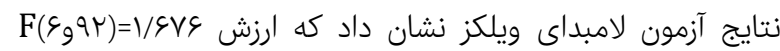

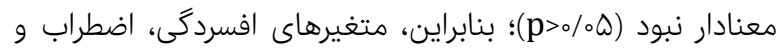

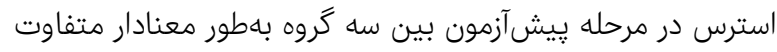

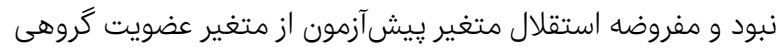

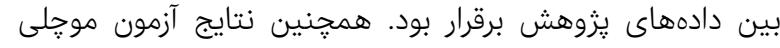

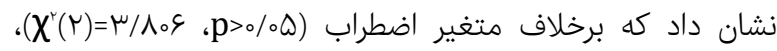

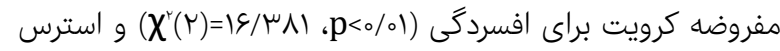

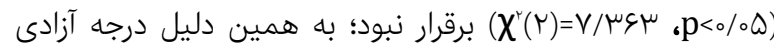

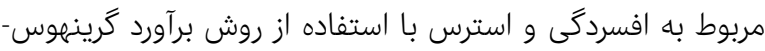

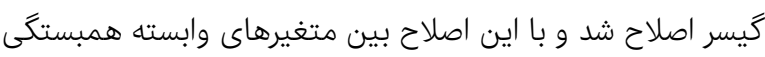

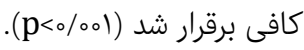

جدول () مقايسه ميانكَين متغيرهاى مقياس DASS-21 بين دو گروه آزمايش و

\begin{tabular}{|c|c|c|c|}
\hline ييكيرى & يسآزمون & ييشآزمون & كروهها \\
\hline & & & افسردگى \\
\hline$N / \varepsilon \mid \pm r / 91$ & $10 / 0 \Lambda \pm \varepsilon / T r$ & $17 / / Y \pm 0 / \circ \varepsilon$ & معنوى-مذهبى \\
\hline$q / \Lambda \Lambda \pm r / 0 。$ & $q / 7 \varepsilon \pm \psi / \varepsilon_{0}$ & $17 / 0 r \pm \mu / q \varepsilon$ & اميددرمانى \\
\hline 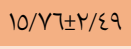 & $17 / \mu_{ \pm} \pm 1 / \wedge 0$ & $1 V / 00 \pm r / 10$ & كنترل \\
\hline & & & اضطراب \\
\hline$\Lambda / \varepsilon V \pm r / \varepsilon V$ & $Q / \Lambda r \pm \varepsilon / O V$ & $1 \varepsilon / V_{\circ} \pm 0 / Y_{0}$ & معنوى-مذهبى \\
\hline 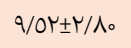 & $9 / \vee 7 \pm \varepsilon / 19$ & $17 / T \Psi \pm \varepsilon / 79$ & اميد درمانى \\
\hline $17 / V\rceil \pm r / \wedge \varepsilon$ & $17 / \circ 0 \pm r / 0 V$ & $|V / r q \pm r /| \mid$ & كنترل \\
\hline & & & استرس \\
\hline$V / \mathcal{E} \pm r / T O$ & $10 /{ }^{\prime} 0 \pm \varepsilon / 01$ & $1 \% / O r \pm \varepsilon / \Lambda \Lambda$ & معنوى-مذهبى \\
\hline$q / \varepsilon \vee \pm r / l r$ & $q / ৭ \varepsilon \pm \psi / q 1$ & $\mid \varepsilon / \Lambda \Lambda \pm \varepsilon / V_{0}$ & اميددرمانى \\
\hline $10 / T_{0} \pm r / 19$ & $10 / V_{0} \pm \Gamma / \tau \mu$ & $\mid \Psi / ৭ \varepsilon \pm 1 / 01$ & كنترل \\
\hline
\end{tabular}

با توجه به نتايج مقياس DASS-21، در مرحله يِيشآزمون همسران جانبازان مبتلا به PTSD نمرات بالايى را از زيرمقياسهاس استراس استرس،

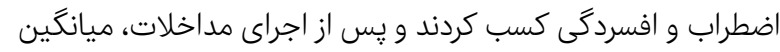

وجودى مبتنى بر ديدكاه ويكتور فرانكل [21,22]، معنوى-مذهبى

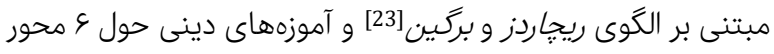

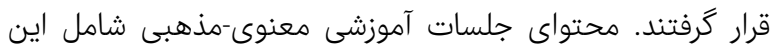

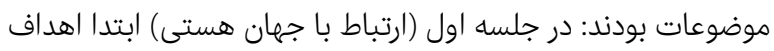

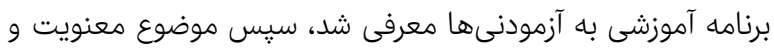

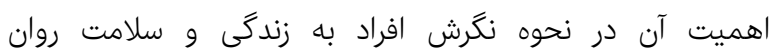

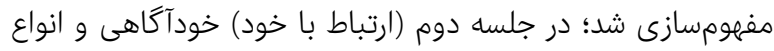

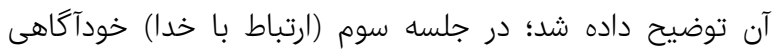

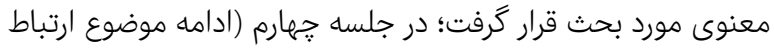

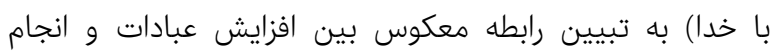

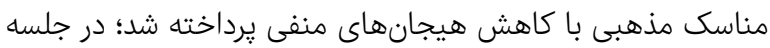

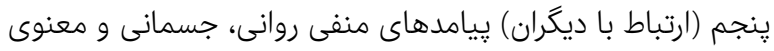

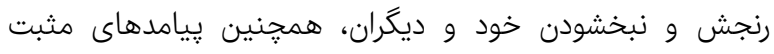

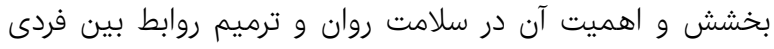

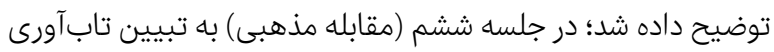

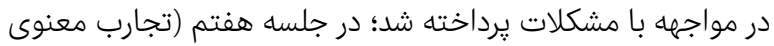

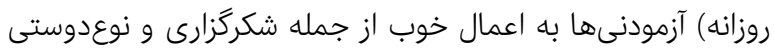

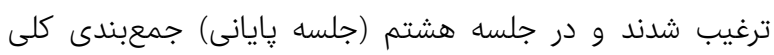

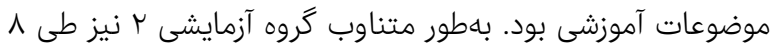

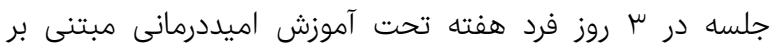

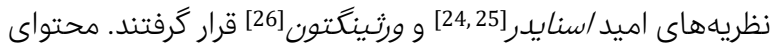
جلسات آموزشى اميددرمانى شامل اين موضوعات بوريدات بودند: در جلسها

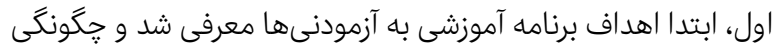

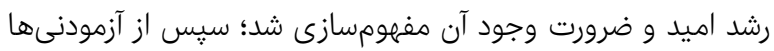

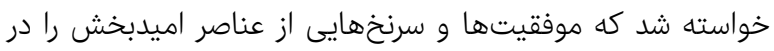

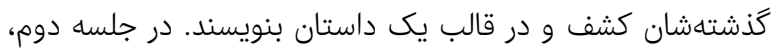

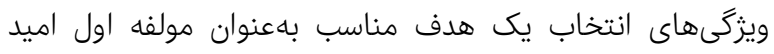

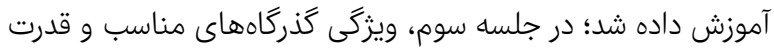

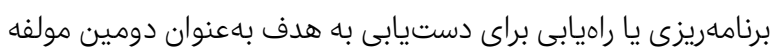

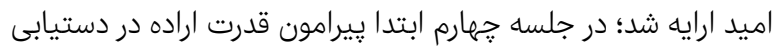

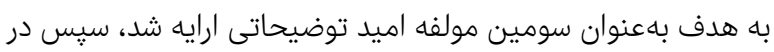

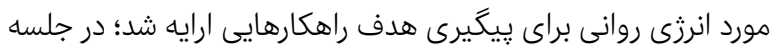

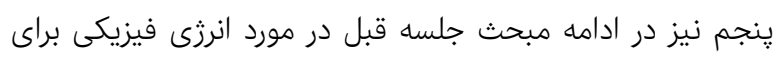

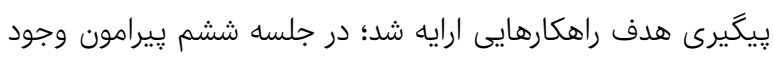

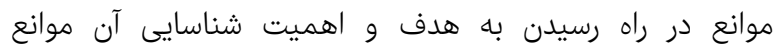

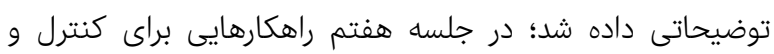

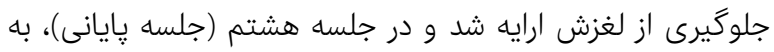

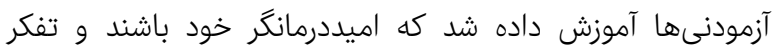

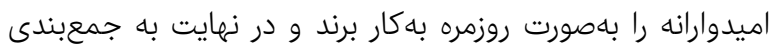

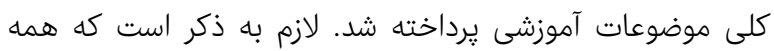

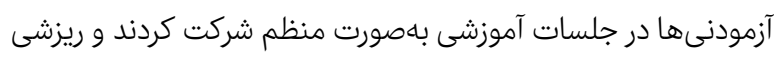

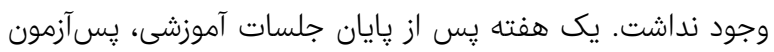

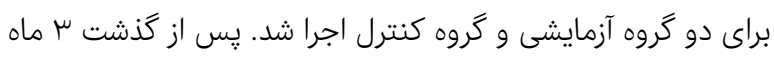

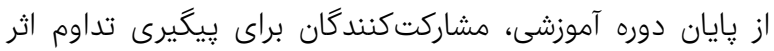




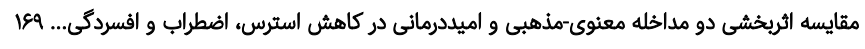

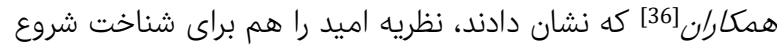

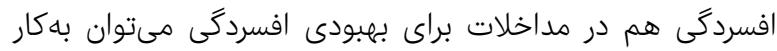

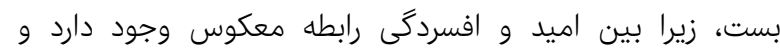

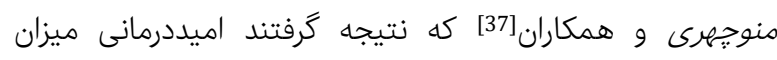
افسردگى را در همسران معتادان كاهش مى هـدهد، همسو است. در

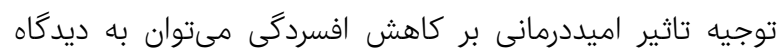

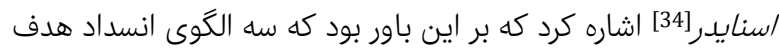

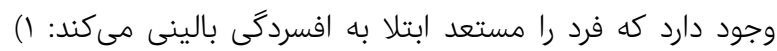

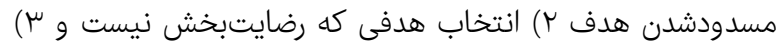

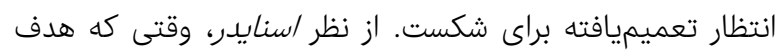

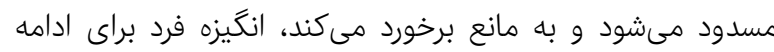

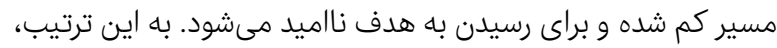

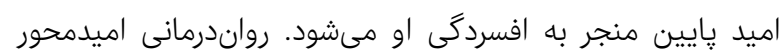

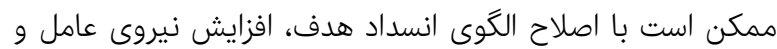

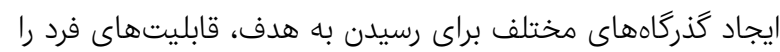

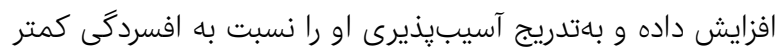
مى كند. برنامه درمانى معنوى-مذهبى منجر به كاهش اضطراب همسران

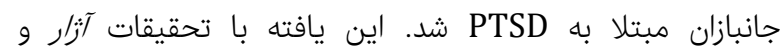

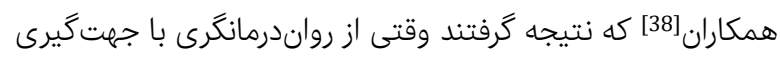
مذهبى استفاده مىشود، نشانههاى اضطراب تعميميافته كاهش

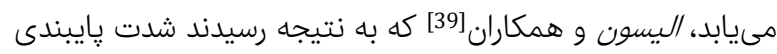

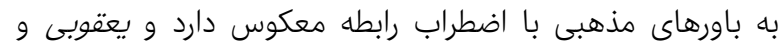

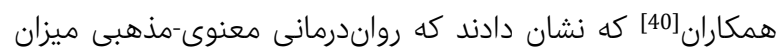
اضطراب دانشجويان را كاهش داده است، همسو است. در توضيح

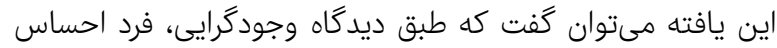

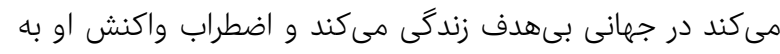
خلأ عظيمى است كه در وجود و معناى زندگى مى مىبيند، اين خلأ

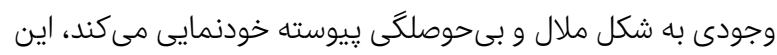

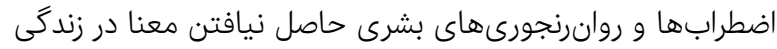

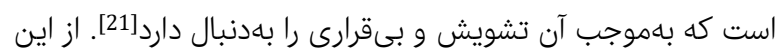

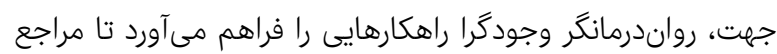

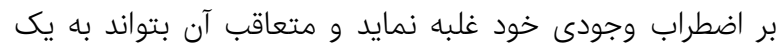

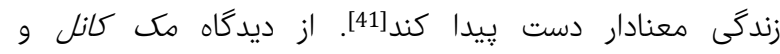
بإرگامنت [27]، اضطراب باد سردرگمى در دين، ترديد معنوى، ارتباط

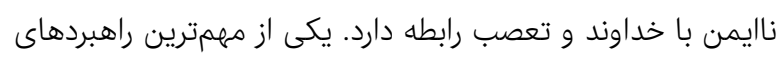

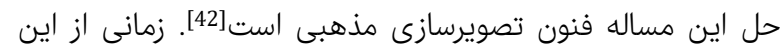

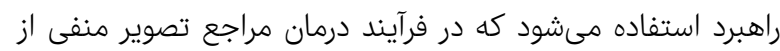

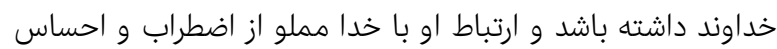

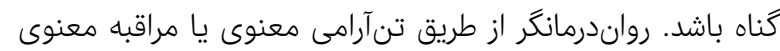
مفهوم واقعى خداوند و حقيقت وجود را در ذهن مراجع اصلاح

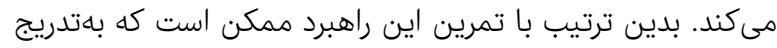

اضطراب فرد كاهش يافته و به آرامش وجودى برسد [23] بـن

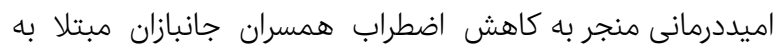

نمرات افسردگى، اضطراب و استرس در گروههاى آزمايش در مراحل

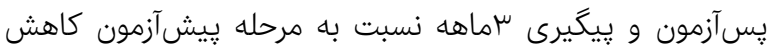

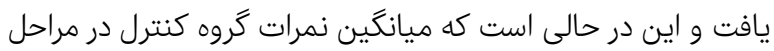

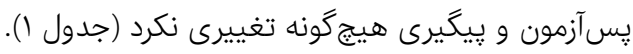
اثر تعاملى دو مداخله معنوى-مذهبى و اميددرمانى معنادار نبود

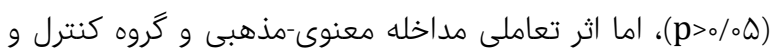

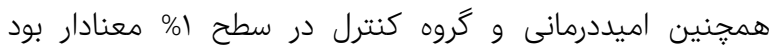

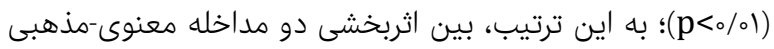

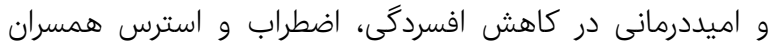

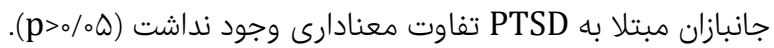

\section{بحث}

يزوهش حاضر با هدف مقايسه اثر دو مداخله معنوى-مذهبى و

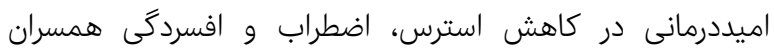
جانبازان مبتلا به PTSD انجام شدردام برنامه درمانى معنوى-مذهبى منجر به كاهش افسردگى همسران

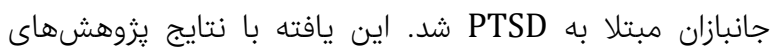

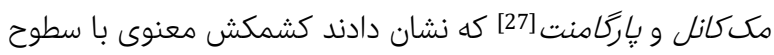

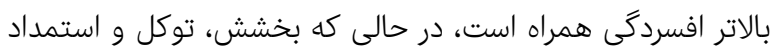
از خداوند با سطوح پايينتر افسردگى همراه است، موريتز

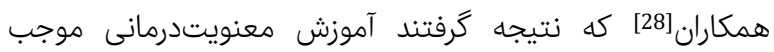

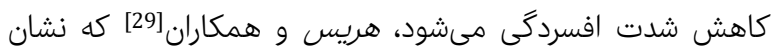

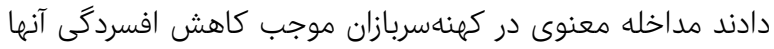

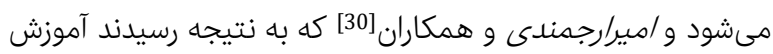
معنويتدرمانى موجب كاهش افسردگى زنان متاهل مى شودرد، همسو

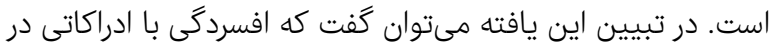

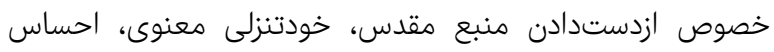

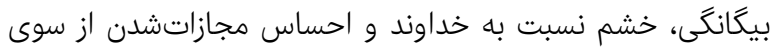

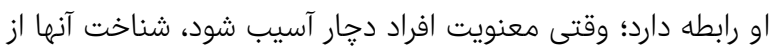
خداوند و وجود دجار اختلال شده و اين شناخت ناصحيح در برداشت

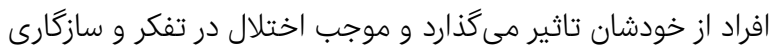

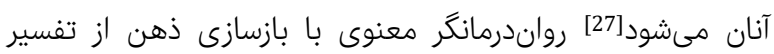

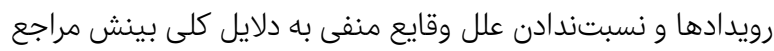

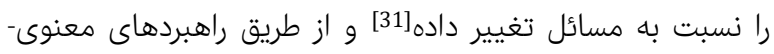

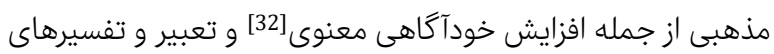
معنوى[23] منابع غنى فطرى مراجع را بيدار كرده و از طريق فراخوانى فردانى

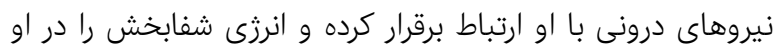

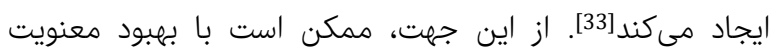

آسيبديده، افسردگى فرد كاهش يابد [31].

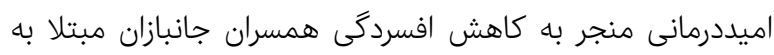

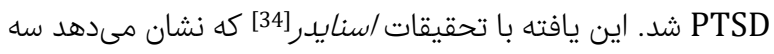

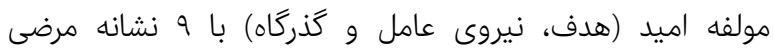

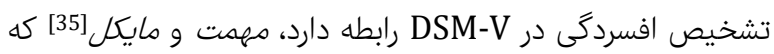

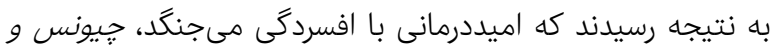


معنوى-مذهبى در انطباق روانى و سازگًارى با شرايط استرسزا و

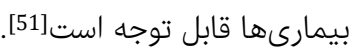
اميددرمانى منجر به كاهش استرس همسران جانيان إنبازان مبتلا به

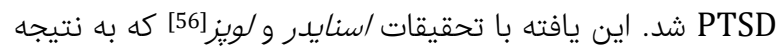
رسيدند اميد وسيلهاى براى يِيشكيرى اوليه و ثانويه از استرس است است

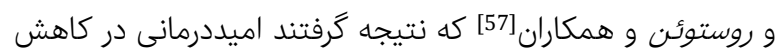

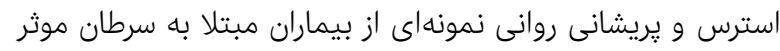

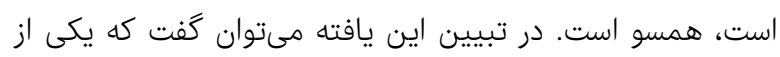

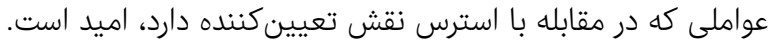

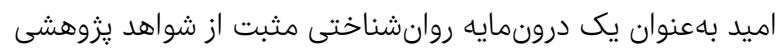

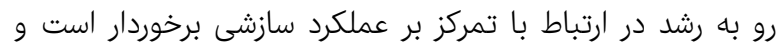

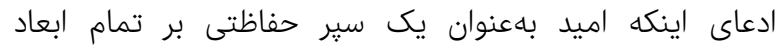

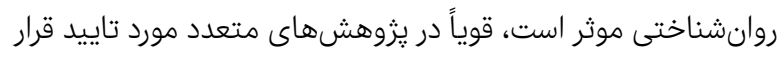

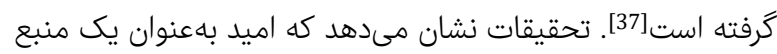

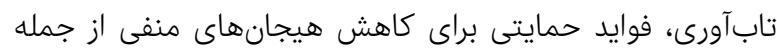

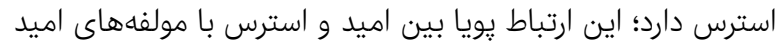

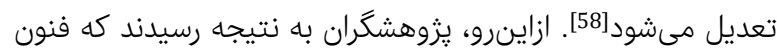

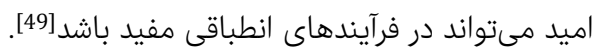

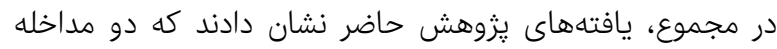

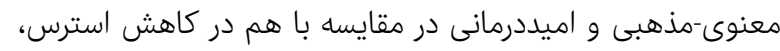

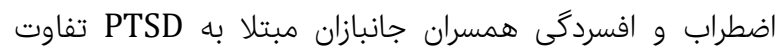

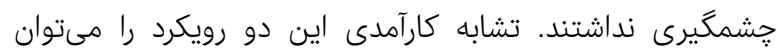

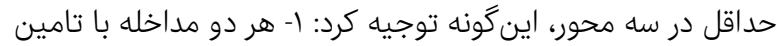

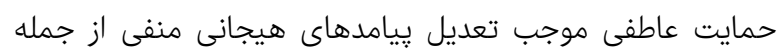

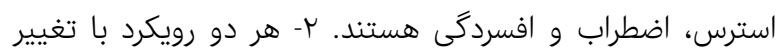

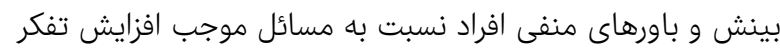

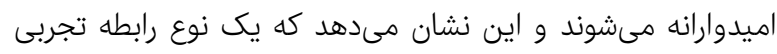

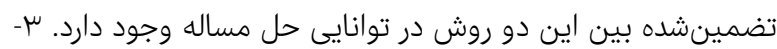

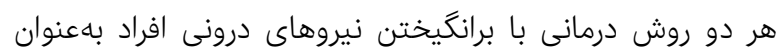

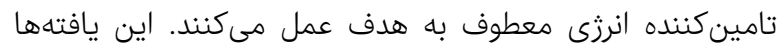

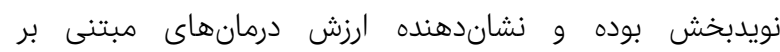

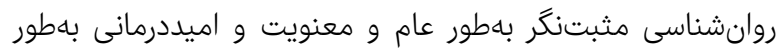

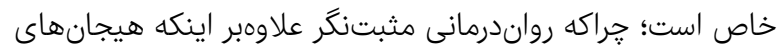

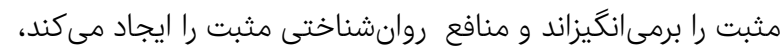

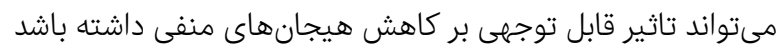
و بهصورت سدى براى وقوع مجدد آنها عمل نمايد.

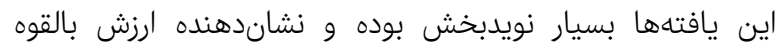

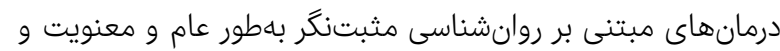

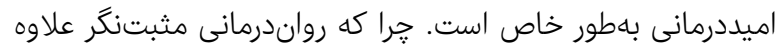

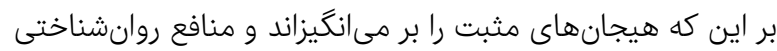

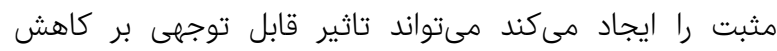

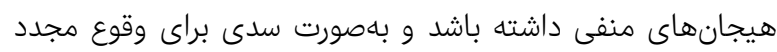

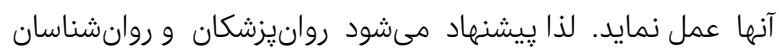

شدSD كرفتند مولفه نيروى عامل اميددرمانى در كاهش نشانههاي اضطراب آنساب و افسردگى كودكان مبتلا به سرطان موثر است. ميشل [44] كه نشان

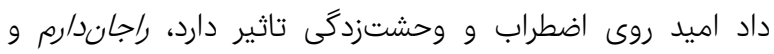

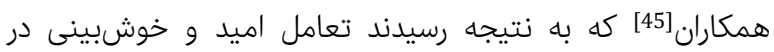

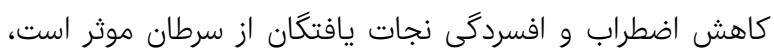

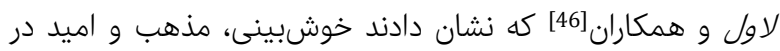

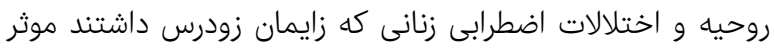

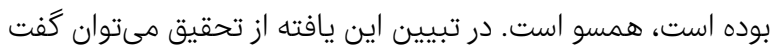

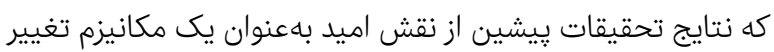

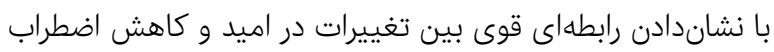

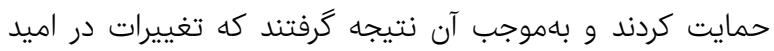

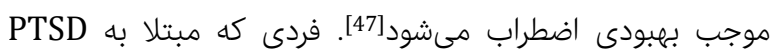

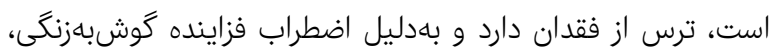

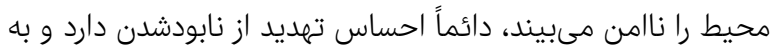

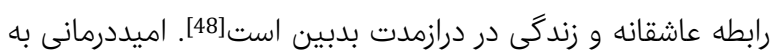

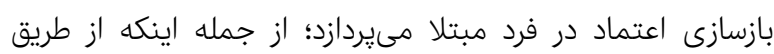

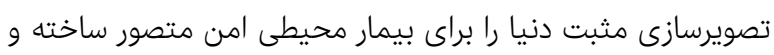

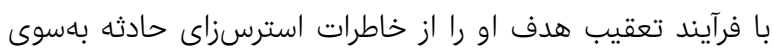

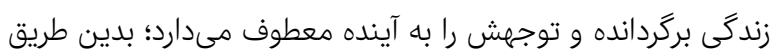

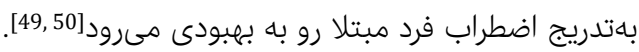

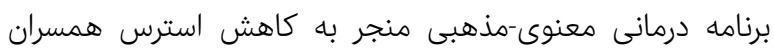

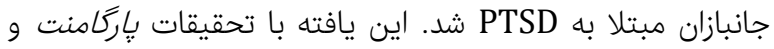

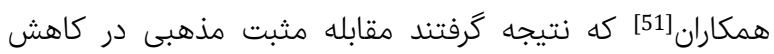

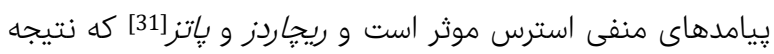

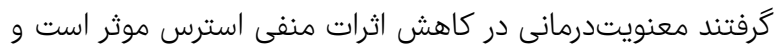

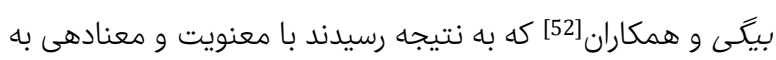

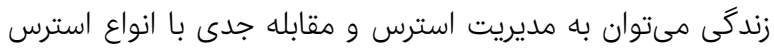

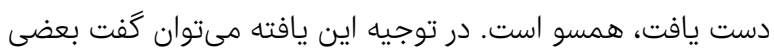

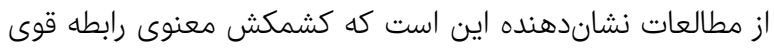

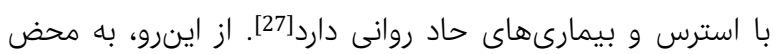

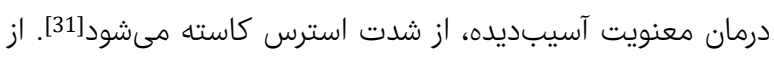

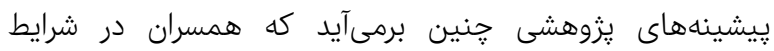

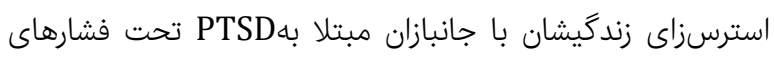

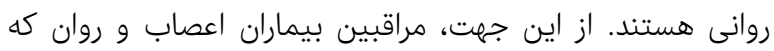

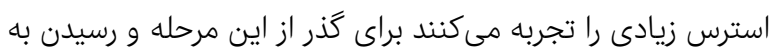

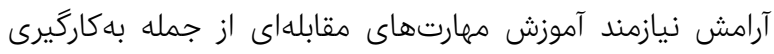

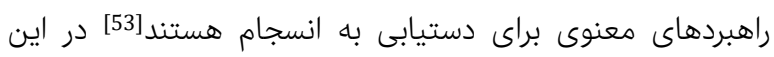

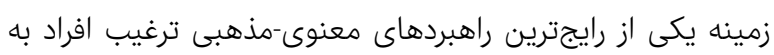

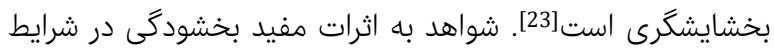

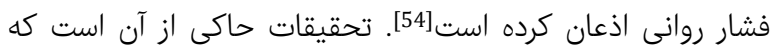

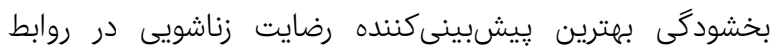

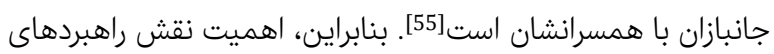


IV مقايسه اثربخشى دو مداخله معنوى-مذهبى و اميددرمانى در كاهش استرس، اضطراب و افسردگى...

7- O'Doonel ML, Creamer M, Pattison P. Post-traumatic stress disorder and depression following trauma: Understanding comorbidity. Am J Psychiatry. 2004;161(8):1390-6.

8- Arzi NB, Solomon Z, Dekel R. Secondary traumatization among wives of veterans with PTSD and posteriorconcussion casualties: Distress, caregiver's burden, and psychological separation. Brain Inj. 2000;14(8):725-36.

9- Klaric M, Franciskovic T, Pernar M, Nembic Moro I, Milicevic R, Cerni Obrdalj E, et al. Caregiver burden and burnout in partners of war veterans with post-traumatic stress disorder. Coll Antropol. 2010;(34 Suppl 1):15-21.

10- Gorman LA, Blow AJ, Ames BD, Reed PL. National guard families after combat: Mental health, use of mental health service, and perceived treatment barriers. Psychiatr Serv. 2011;62(1):28-34.

11- Ahmadi K, Reshadatijou M, Karami G Comparison of depression, anxiety and stress in chemical victims with healthy people in Sardasht. J Babol Univ Med Sci. 2008;12(1):44-50. [Persian]

12- Mikulineer M, Florian V, Solomon Z. Marital intimacy, family support, and secondary traumatization: A study on wives of veterans with and anxiety reactions. Anxiety Stress Coping. 1995;8(3):203-13.

13- Rafieepour A, Farahani S, Mazloomal-Hosseini N, Eshagi Moghadam F. The relationship between personality traits and the desire for divorce in disabled veterans: The mediating role of post-traumatic stress. Sci J Mil Psychol. 2020;10(40):33-44. [Persian]

14- Turgoose D, Murphy D. A systemic review of interventions for supporting partners of military veterans with PTSD. J Mil Veteran Fam Health. 2019;5(2):195-208. 15- Abolmaali K, Aghaeepour-Ghoasaraee M, Kamal A. Comparison of the effectiveness of cognitive-behavioral techniques training and schema-based training on modulating the symptoms of secondary trauma in the spouses of veterans with post-traumatic stress disorder. Jundishapour Med Sci J. 2014;15(2):187-200. [Persian]

16- Kamal A, Abolmaali K. The effectiveness of instruction based on schema therapy in reducing STSD. Iran J War Public Health. 2015;7(1):21-8. [Persian]

17- Bride BE, Robinson MM, Yegidis D, Figley CR. Development and validation of the secondary traumatic stress scale. Res Soc Work Pract. 2004;14(1):27-35.

18- Ahmadi K, Rezapour Y, Davoodi F, Saberi M. Investigate of validity and reliability of secondary trauma stress scale for evaluation of PTSD Symptoms in samples of warfare victims' wives. Iran J War Public Health. 2013;5(3):47-57. [Persian]

19- Lovibond PF, Lovibond SH. The structure of negative emotional states: Comparison of the depression anxiety stress scale (DASS) with the Beck depression and anxiety inventories. Behav Res Ther. 1995;33:335-43.

20- Sahebi A, Asghari MJ, Salari RS. Reliability and validity of the depression anxiety stress scale (DASS-21) for Iranian population. Q Period Iran Psychol. 2005;1(4):3654. [Persian]

21- Frankl VE. Psychiatry and man's quest for meaning. J Relig Health. 1962;1:93-103.

22- Frankl VE. Logotherapy and existentialism. Psychother Theor Res Pract. 1967;4(3):138-42.

23- Richards PS, Bergin AE. Casebook for a spiritual strategy in counselling and psychotherapy. Washington: American Psychiatric Association; 2004.

24- Snyder CR. Target Article: Hope Theory: Rainbows in the mind. Psychol Inquiry J. 2002;13(4):249-75.

25 - Snyder CR. Hand book of hope: Theory, measures and

$$
\begin{aligned}
& \text { جهت كمك به بهبود علائم همسران جانبازان از نتايج يزوهش حاضر }
\end{aligned}
$$

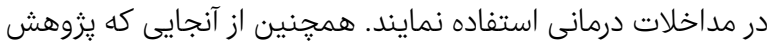

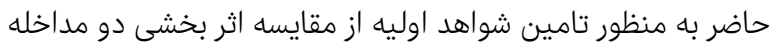

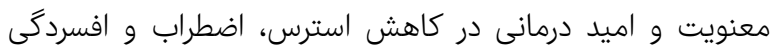

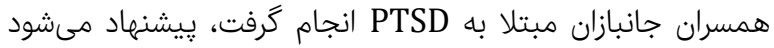

$$
\begin{aligned}
& \text { يزوهشهاى آتى مجدداً اثر بخشى اين دو مداخله را روى همسران إنهان }
\end{aligned}
$$

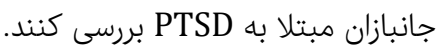

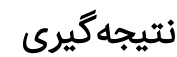

هر دو روش مداخله معنوى-مذهبى و اميددرمانى بهعنوان مداخلات

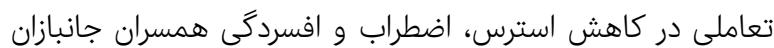
مبتلا به PTSD موثرند و در مقايسه با هم تفاوتى ندارند.

$$
\begin{aligned}
& \text { تشكر و قدردانى: بدينوسيله از همسران جانبازان مبتلا به PTSD كه با }
\end{aligned}
$$

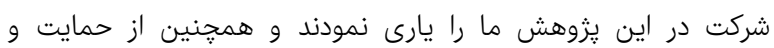

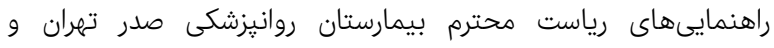

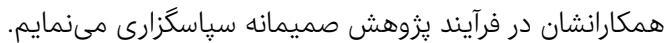

$$
\begin{aligned}
& \text { تاييديه اخلاقى: موردى بيان نشد. تُران. } \\
& \text { تعارض منافع: تعارض منافعى وجود نداردي }
\end{aligned}
$$

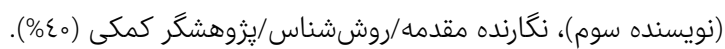

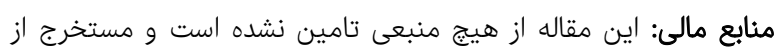

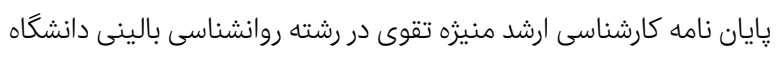

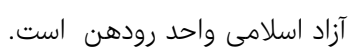

منابع

1- Kazemi AS, Banijamali SS, Ahadi H, Farrokhi NA. Comparison of the effectiveness of two training strategies- cognitive behavioral and mindfulness in reduction of STSD symptoms and psychological problems of devotee's wives with PTSD. Med Sci J Islam Azad Univ. 2009;22(2):122-9. [Persian]

2- American psychiatric association. Diagnostic and statistical manual of mental disorders. Washington: American Psychiatric Association; 2013.

3- Galvoski T, Lyons JA. Psychological sequelae of combat violence: A review of the impact of PTSD on the veteran's family and possible interventions. Aggres Violent Behav. 2004;9(5):477-501.

4- Jenkins SR, Baird S. Secondary traumatic stress and vicarious trauma: A validational study. J Traumatic Stress. 2002;15(5):423-32.

5- Klaric M, Franciskovic T, Obrdalj EC, Petric D, Britvic D, Zovko N. Psychiatric and health impact of primary and secondary traumatization in wives of veterans with posttraumatic stress disorder. Psychiatr Danub. 2012;24(3):280-6.

6- Harvey A, Bryant R. The relationship between acute stress disorder and posttraumatic stress disorder: A prospective evaluation of motor vehicle accident survivors. J Consult Clin Psychol. 1998;66(3):507-12. 
2000.

43- German JN, Leonard D, Heath CL, Stewart SM, Leavey PJ. Hope as a predictor of anxiety and depressive symptoms following pediatric cancer diagnosis. J Pediatr Psychol. 2018;43(2):152-61.

44- Michael ST. Hope conquers fear: Overcoming anxiety and panic attacks. Handb Hope. 2000;301-19.

45- Rajandram RK, MY HO S, Samman N, Chan N, Mc Grath C, Zwahlen RA. Interaction of hope and optimism with anxiety and depression in a specific group of cancer survivors: A preliminary study. BMC Res Notes. 2011;4:519.

46- Lowell EP, Tonnsen BL, Bailey DB, Roberts JE. The effects of optimism, religion, and hope on mood and anxiety disorders in women with the FMR1 premutation. J Intellect Disabil Res. 2017;61(10):916-27.

47- Gallagher MW, Long LJ, Richardson A, Dsouza J, Boswell JF, Farchion TJ, et al. Examining hope as a trans diagnostic mechanism of change across anxiety disorders and CBT treatment protocols. Behav Ther. 2020;51(1):190-202.

48- Harvey MR. An ecological view of psychological trauma and recovery. J Trauma Stress. 1996;9(1):3-23.

49-Synder CR, Lehman KA, Kluck B, Monsson Y. Hope for rehabilitation and vice versa. Rehabilit Psychol. 2006;51(2):89-112.

50- Lopez SJ, Floyd RK, Ulven JC, Snyder CR. Hope therapy: Helping clients build a house of hope. In: Snyder CR, editor, Handbook of hope: Theory, measures, and applications. Unknown city: Academic Press; 2000.

51- Pargament KI, Smith BW, Koenig HG, Perez L. Patterns of positive and negative religious coping with major life stressors. J Sci Stud Relig. 1998;37(4):710-24.

52- Beigi A, Mohammadifar MA, Eisa Morad Rudbaneh A, Najafi $M$. The role of spirituality in predicting coping styles with stress. J Couns Cult Psychother. 2013;4(14). [Persian]

53- Pearce MJ, Medoff D, Lawrence RE. Religious coping among adults caring for family members with serious mental illness. Community Ment Health J. 2016;52(2):194-202.

54- Worthington EL, Scherer M. Forgiveness is an emotion-focused coping strategy that can reduce health risks and promote health and resilience: Theory, review, and hypotheses. Psychol Health. 2004;19(3):385-405. 55- Ehteshamzadeh P, Mekvandi B, Bagheri A. Relationship among forgiveness, perfectionism, and intimacy with marital satisfaction in military veterans and their spouses. Journal of Social Psychology (New Findings In Psychology). 2009;4(12):123-36.

56- Snyder CR, Lopez SJ. Handbook of positive psychology. Oxford: Oxford University Press; 2002.

57- Rustoen T, Cooper BA, Miaskowski C. A longitudinal study of the effects of a hope intervention on Levels of hope and psychological distress in a community-based sample of oncology patients. Eur J Oncol Nurs. 2011;15(4):351-7.

58- Antony DO, Edwards LM, Bergman CS. Hope as a source of resilience in later adulthood. Personal Individ Differ. 2006;41(7):1263-73. applications. Cambridge: Academic Press; 2000.

26- Worthington EL, Hight TL, Ripley JS, Perrone KM, Kurusu TA, Jones DR. Strategic hopefocused relationshipenrichment counseling with individual couples. J Couns Psychol. 1997;44:381-89.

27- McConnell KM, Pargament KI. Examining the links between spiritual struggles and symptoms of psychopathology in a national sample. J Clin Psychol. 2006;62(12):1469-84.

28- Moritz S, Kelly MT, Xu TJ, Rickhi B. A spirituality teaching program for depression: Qualitative finding on cognitive and emotional changes. Complement Ther Med. 2011;19(4):201-7.

29- Harris JI, Erbes CR, Engdahl BE, Thuras P, MurraySwank N, Grace D, et al. The effectiveness of spiritually integrated intervention in veterans exposed to trauma. J Clin Psychol. 2011;67(4):425-38.

30- Amirarjmandi SZ, Hashemian K, Niknam M. Effect of spiritual group training on decreasing depression and increasing marital satisfaction among married woman in Tehran, region 5. Educ Psychol. 2015;11(35):137-58. [Persian]

31- Richards PS, Potts RW. Using spiritual interventions in psychotherapy: practices, successes, failures and ethical concerns of Mormon psychotherapy. Prof Psychol Res Pract. 1995;26(2):163-70.

32- Bolhari J, Mohsenikabir M. Compiling and evaluating educational package of spiritual skills for university students. Iran J Psychiatry Clin Pschol. 2019;25(2):21021. [Persian]

33- Taylor PB, Ferszt GG. Spiritual healing. Holist Nurs Pract. 1990;4(4):32-8.

34- Snyder CR. Hope and depression: A light in the darkness. J Soc Clin Psychol. 2004;23(3):347-51.

35- Mehmet C, Michael R. You have got hope, studies show "hopetherapy" fights depression. Atlanta: New SRX Science; 2009.

36- Cheavens JS, Feldman DB, Gum A, Michael ST, Snyder CR. Hope therapy in a Community sample: A pilot investigation. Soc Indicators Res. 2006;77(1):61-78.

37- Manouchehri M, Golzari M, Kordmirza-Nikoozadeh E. The effectiveness of hope therapy training on the use of positive coping methods with stress in women with addicted spouses. Q Clin Psychol Stud. 2015;6(21):78-90. [Persian]

38- Azhar MZ, Varma SL, Dharap AS. Religious psychotherapy in anxiety disorder patients. Acta Psychiatr Scand. 1994;90(1):1-3.

39- Elison CG, Burdette AM, Hill TD. Blessed assurance: Religion, anxiety, and tranquility among U.S. adults. Soc Sci Res. 2009;3(38):656-67.

40- Yagoobi H, Sohrabi F, Mohammadzadeh A. The comparison of cognitive-behavior therapy and Islamic based spiritual-religion psychotherapy on reducing of students overt anxiety. J Res Behav Sci. 2012;10(2).

41- Wong PTP. Meaning therapy: An integrative and positive psychotherapy. J Contemp Psychother. 2010;40(2):85-93.

42- West W. Psychotherapy and spirituality: Crossing the line between therapy and religion. Thousand Oaks: SAGE; 